\title{
Quantitative evaluation of axial wall taper in prepared artificial teeth
}

\author{
Yayoi Okuyama, Shin Kasahara and Kohei Kimura \\ Division of Fixed Prosthodontics, Department of Restorative Dentistry, \\ Tohoku University Graduate School of Dentistry, Sendai, Japan
}

(Received 4 May and accepted 2 September 2005)

\begin{abstract}
The purpose of this study was to quantitatively evaluate the axial wall taper of prepared artificial teeth using a non-contact three-dimensional shape measuring system. A total of 54 artificial teeth prepared by pre-clinical dental students for complete cast restorations were evaluated. For quantitative analysis, five cross sections were computer-graphically placed perpendicularly to the $z$-axis. The surface coordinate values $(x, y, z)$ of each cross section were converted into polar coordinate values $(\mathbf{r}, \theta)$, which were then graphically rendered to a two-dimensional plane. At four points, each $90^{\circ}$ from the distal center point of the cross section, the axial wall taper was quantitatively calculated using a formula based on the differences in radius between the highest and lowest positions of the cross sections of the prepared tooth. The average calculated taper was $5.8^{\circ}$ in the distal region, $21.7^{\circ}$ in the buccal region, $14.9^{\circ}$ in the mesial region and $12.5^{\circ}$ in the lingual region. These results suggest that the axial wall taper of prepared teeth can be quantitatively evaluated using this measuring system. (J. Oral Sci. 47, 129-133, 2005)
\end{abstract}

Keywords: quantitative evaluation; axial wall taper; tooth preparation; non-contact measuring system; objective evaluation.

Correspondence to Dr. Yayoi Okuyama, Division of Fixed Prosthodontics, Tohoku University Graduate School of Dentistry, 4-1 Seiryo-machi, Aoba-ku, Sendai, 980-8575, Japan

Tel: +81-22-717-8363

Fax: +81-22-717-8367

E-mail: yayoi-o@mail.tains.tohoku.ac.jp

\section{Introduction}

Tooth preparation for complete cast restorations is a routine clinical procedure performed intra-orally prior to taking the impression. Pre-clinical students require training in the preparation technique, and quantitative evaluation of the axial wall taper of prepared artificial teeth permits students to evaluate their own skill level.

Applications of computer-aided systems to restoration procedures have been published. Three representative computer-aided design/computer-aided manufacturing (CAD/CAM) techniques were recently introduced (1). The Cerec computer-aided design/computer-integrated manufacturing (CAD/CIM) systems (2-5) and other improved systems use an optical impression technique combined with an in situ grinding procedure. Another system employs a laser displacement meter together with double sensors $(6,7)$. Engineering applications of automation are being increasingly integrated into the fabrication of dental prostheses (1-5). Although several reports on the measurement of the axial wall taper of abutment teeth have been published (8-10), their techniques include neither computerized evaluation nor automatic calculation.

In recent years, inlay cavities and teeth prepared for complete cast restorations have been quantitatively evaluated using a computer-aided system. CLINSIM (Morita, Tokyo, Japan) has been used for pre-clinical laboratory simulation. Kournetas N. et al (11) reported that PREPassistant (KaVo Dental GmbH, Biberach/Riss, Germany), the latest system by KaVo for 3-dimensional surveying in dental education, could scan one prepared tooth in 2 minutes or less and its measurement accuracy was 0.020 $\mathrm{mm}$. Its accuracy and repeatability were acceptable for practical purposes (11). DentSim Compact (Yoshida, 
Tokyo, Japan) has been developed as an advanced simulation system for the instruction of dental students in pre-clinical restorative procedures. First-year students, who received most of the preparative operative training using this system, learned faster than and arrived at the same level of performance as when conventional laboratories were used (12).

The objective of this study was to quantitatively evaluate the axial wall taper of prepared artificial teeth using a non-contact three-dimensional shape measuring system. This report describes the computerized calculation method designed for the visualization of the axial wall taper of abutment teeth immediately after tooth reduction.

\section{Materials and Methods}

A total of 54 artificial teeth (A5A-500, Nissin, Kyoto, Japan) prepared by dental students for complete cast restorations were evaluated. These students had completed a fixed prosthodontics course, but had no clinical experience. Students prepared the maxillary left first molar on a dental cast with artificial gingiva mounted in a manikin head. For tooth preparation, a high-speed five-diamond points (Diamond Point FG, No. 201, 202, 204, F204 and 340; ISO \#016, 021, 013, 012 and 018; Shohu, Kyoto, Japan) and a stone point (Carborundum Point FG No. 24; Shohu, Kyoto, Japan) with water coolant were used. Each student was instructed to perform reduction with 1.0 - 1.5 $\mathrm{mm}$ occlusal clearance and 2-5 degree axial taper. Fig. 1 illustrates the tooth reduction requisite for this design.

The shape of the prepared tooth was measured using a

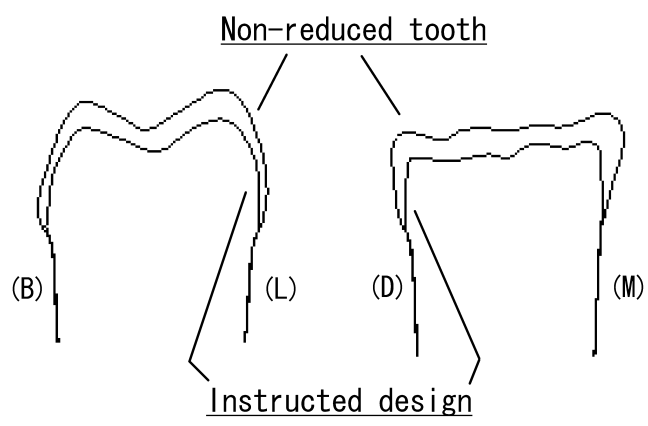

(B) : Buccal side

(L) : Lingual side

(M) :Mesial side

(D) :Distal side

Fig. 1 Superimposed configurations of instructed reduction design and non-reduced artificial tooth.

A: Mesial view, B: Buccal view

Both configurations show the cross sections through the central fossae. non-contact semiconductor laser beam three-dimensional shape measuring system (Surflacer VMS-250R-H, Unisn, Osaka, Japan) with a minimum resolution of $0.035 \mathrm{~mm}$. This system consists of a main measuring system unit, an image processor board and a DOS/V PC based controller. The fundamental principle of this measuring system is a trigonometric survey with the slit-projection method. The main measuring system unit consisted of a slit-ray projector, two CCD cameras to read the image, $\mathrm{X}$ and $\mathrm{Y}$ tables and an $\mathrm{R}$ table with original mounting jig which rotated the object to make split measurements around its circumference. The slit-ray projector projected a beam of light from a semiconductor laser (wave length: $670 \mathrm{~nm}$, output: $3 \mathrm{~mW}$ ). An object on the mounting jig was adjusted by moving the $\mathrm{X}$ table to the slit-ray projection area and by rotating the $\mathrm{R}$ table to improve the accuracy for calculation. One scan line was measured per second and the data from 256 points per scan line were processed simultaneously. The origin of the coordinate axes of this measuring system unit was as follows; $\mathrm{x}=0$ represented the scanning starting point, $\mathrm{y}=0, \mathrm{z}=0$ represented the center position of rotation.

After spraying the prepared tooth with developer (Color Check FD-U, Taseto, Kawasaki, Japan) in order to reduce the artificial tooth surface gloss, the shape of the prepared tooth was determined. To compensate for unmeasured regions, the prepared tooth was measured from two directions (occlusal and axial wall direction) on the original mounting jig and the data were combined with 3D Graphics Software (Imageware9, EDS PLM Solutions, Detroit,

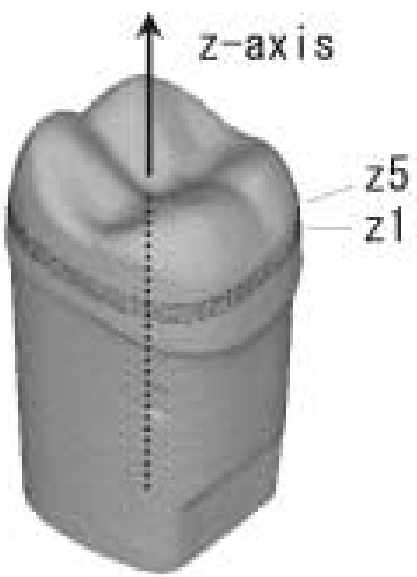

Fig. 2 Mesio-lingual view of a computer-graphic surface model of a prepared artificial tooth with five cross sections.

The direction of the $\mathrm{z}$-axis was at right angles to the artificial tooth bottom. Five cross sections were displayed on the axial wall. The highest position is labeled $\mathrm{z}_{5}$, and the lowest position $\mathrm{z}_{1}$. 
USA) to yield a profile of the whole surface. It was then possible to visualize computer-graphically the entire shape of the prepared tooth from any direction (Fig. 2). The axial measuring pitches were $0.1 \mathrm{~mm}$ for the $\mathrm{x}$-axis, 0.12 $\mathrm{mm}$ for the $\mathrm{y}$-axis and $0.05 \mathrm{~mm}$ for the $\mathrm{z}$-axis.

For quantitative analysis, five cross sections were made perpendicular to the z-axis (at intervals of $0.2 \mathrm{~mm}$ ) (Fig. 2 ). The surface coordinate values $(\mathrm{x}, \mathrm{y}, \mathrm{z})$ of each cross section on the prepared tooth were extracted from all the measured coordinate values. These rectangular coordinate values $(\mathrm{x}, \mathrm{y}, \mathrm{z})$ were converted into polar coordinate values $(\mathrm{r}, \theta)$ (Fig. 3). The polar coordinate values were then graphically rendered to a two-dimensional plane. Thus, it was possible to see the preparation result as a five-curve shape. By observing the curves, the axial wall taper of each prepared tooth was quantitatively evaluated. The axial wall taper based on the differences in radius between $\mathrm{z} 1$ and z5 was calculated with the following formula:

axial wall taper $=\alpha$

$\tan \alpha=\Delta \mathrm{r} /(\mathrm{z} 5-\mathrm{z} 1) \quad$ where $\Delta \mathrm{r}=\mathrm{r} 1-\mathrm{r} 5, \mathrm{z} 5-\mathrm{z} 1=0.8$

Radius values where $\theta$ was closest to $0^{\circ}, 90^{\circ}, 180^{\circ}$ and $270^{\circ}$ were selected as the representative data. The point of $\theta=0^{\circ}$ represented the distal center point, $\theta=90^{\circ}$ represented the buccal center point, $\theta=180^{\circ}$ represented the mesial center point, and $\theta=270^{\circ}$ represented the lingual center point.

At these four center points, the mean differences and standard deviation (SD) in radius between the highest and lowest positions of the cross section and the average taper and SD were calculated.

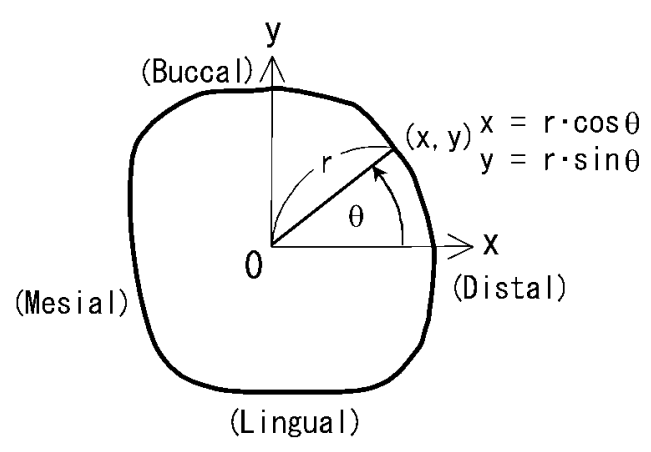

Fig. 3 Schematic of the occlusal view of the cross section of a prepared artificial tooth.

The point of $\theta=0^{\circ}$ represents the distal center point, and the point of $\theta=90^{\circ}$ represents the buccal center point. The converted calculation was carried out following an arrow $(\theta)$, rotated in a counterclockwise direction from the distal center point.

\section{Results}

By displaying graphic renderings of the polar coordinate values on a two-dimensional plane, it was possible to visualize the preparation results as a five-curve shape. These five curves showed various patterns, according to the preparation results: overlapping, parallel, scattered and intersecting.

Table 1 shows the mean differences and SD in radius between the highest and lowest positions of the cross sections of the prepared tooth. The greatest dispersion of the differences $(0.33 \mathrm{~mm})$ was obtained in the buccal region, and the least dispersion of the differences $(0.08 \mathrm{~mm})$ was in the distal region.

It was possible to quantitatively calculate the axial wall taper using a formula based on the differences in radius between the highest and lowest positions of the cross sections of the prepared tooth. Table 2 summarizes the results of the average calculated taper at the same center points shown in Table 1. The highest average calculated taper $\left(21.7^{\circ}\right)$ was obtained in the buccal region, and the lowest average calculated taper $\left(5.8^{\circ}\right)$ was recorded in the distal region. The SD of the calculated tapers in the mesial and distal regions were smaller than those of buccal and lingual region.

\section{Discussion}

A novel method has been developed for automatically calculating the axial wall taper of a prepared artificial tooth, using a non-contact three-dimensional shape measuring system. This method is based on the concept

Table 1 Differences in radius between the highest and lowest positions of the cross sections

\begin{tabular}{cccccc}
\hline & Mean & SD & Minimum & Maximum & $\mathrm{n}$ \\
\hline Mesial & 0.22 & 0.11 & 0.04 & 0.63 & 46 \\
Distal & 0.08 & 0.09 & -0.11 & 0.40 & 52 \\
Buccal & 0.33 & 0.17 & -0.02 & 0.93 & 54 \\
Lingual & 0.19 & 0.17 & -0.06 & 1.05 & 53 \\
\hline
\end{tabular}

(mm)

Table 2 Calculated taper

\begin{tabular}{cccccc}
\hline & Mean & $\mathrm{SD}$ & Minimum & Maximum & $\mathrm{n}$ \\
\hline Mesial & 14.9 & 6.7 & 2.6 & 38.3 & 46 \\
Distal & 5.8 & 6.2 & -7.8 & 26.7 & 52 \\
Buccal & 21.7 & 9.4 & -1.3 & 49.2 & 54 \\
Lingual & 12.5 & 9.8 & -4.2 & 52.8 & 53 \\
\hline
\end{tabular}

(degrees) 
that perpendicular cross sections are made on the long-axis of the prepared artificial tooth (Fig. 2).

The silhouettes of prepared teeth have routinely been traced on tracing paper, with lines drawn parallel to the traced axial walls at the gingival positions and extended until they met at an angle, identified as the convergence angle, which was measured manually with a protractor (810). Half the value of this angle was equal to the "taper" $(9,10)$. Selected or interpolated data from the traced contours of prepared teeth were used in these conventional methods. With the method described in the present study, the actual measured data of the prepared teeth were used in order to calculate the axial wall taper with increased accuracy.

On preliminary examination, the calculated mean measurement accuracy was $0.04 \pm 0.01 \mathrm{~mm}(\mathrm{n}=5)$, and the taper differences were $0.04 \pm 0.03^{\circ}(n=5)$. These results confirmed that this measuring system had sufficient accuracy for quantitative evaluation of axial wall taper of artificial teeth prepared for complete cast restorations.

Furthermore, the features of the prepared teeth are clearly recognized by drawing five curves on a twodimensional plane. In cases of poor preparation, for example, the five curves cross in various regions and the degrees of taper are negative. With excessive occlusal reduction, the five curves are dispersed widely, because they are located not on the axial wall but in the occlusal region. Even when the five cross sections are located in the occlusal region, or on the unreduced artificial teeth, the axial wall taper is calculated automatically. Therefore, a tooth with excessive occlusal reduction shows quite a large degree of taper $\left(73.8^{\circ}\right)$ in the lingual region. It is essential that computer-graphics are used to determine whether the five cross sections are located on the axial wall. In this study, the teeth in which the five cross sections were located out of the axial wall were avoided. As a result, the number of the calculated samples varied at each center point.

For reference, the average taper calculated mesio-distally was $10.4 \pm 6.5^{\circ}$, and the average taper calculated buccolingually was $17.1 \pm 9.6^{\circ}$. The average calculated taper degrees and SD in the mesio-distal region were smaller than those of the bucco-lingual region since mesio-distal preparation is generally performed with close attention so as not to damage the adjacent teeth. In addition, the longaxis of the artificial tooth was inclined approximately $7^{\circ}$ to the buccal side in the dental cast and the students did not notice the tooth inclination.

Using the method described in this paper, the inclination between minute z-distances can be calculated (Fig. 2). The calculated tapers of the instructed reduction form (Fig. 1, 2) were $4.2^{\circ}, 1.3^{\circ}, 13.4^{\circ}$ and $4.2^{\circ}$ at the mesial, distal, buccal and lingual center points, respectively. The buccal data suggest that the five cross sections are located at a slightly high position, that is, on two-planes of the axial wall. This study suggests that appropriate z-distances are linked directly to the calculation of the axial wall taper.

Subjective evaluation of the abutment teeth prepared by dental students is usually carried out. However, this noncontact three-dimensional shape measuring system showed that objective evaluation of abutment teeth is possible. Objective evaluation should be all-inclusive; that is, it should be combined with quantitative evaluation of other parameters - volume of occlusal reduction, margin shape and surface smoothness. Instructors should teach their students objectively with the help of both quantitatively accurate data and evaluation. In this regard, the system described here holds promise.

\section{References}

1. Rekow D (1987) Computer-aided design and manufacturing in dentistry: a review of the state of the art. J Prosthet Dent 58, 512-516

2. Mörmann WH, Bindl A (1996) The new creativity in ceramic restorations: dental CAD-CIM. Quintessence Int 27, 821-828

3. Mou SH, Chai T, Wang JS, Shiau YY (2002) Influence of different convergence angles and tooth preparation heights on the internal adaptation of Cerec crowns. J Prosthet Dent 87, 248-255

4. Nakamura T, Dei N, Kojima T, Wakabayashi K (2003) Marginal and internal fit of Cerec 3 CAD/CAM all-ceramic crowns. Int J Prosthodont $16,244-248$

5. Bindl A, Mörmann WH (2005) Marginal and internal fit of all-ceramic CAD/CAM crown-copings on chamfer preparations. J Oral Rehabil 32,441-447

6. Kimura H, Sohmura T, Watanabe T (1990) Three dimensional shape measurement of teeth. (Part 3) On the measurement by the newly developed laser displacement meter with double sensors. J Osaka Univ Dent Sch 30, 31-37

7. Sohmura T, Takahashi J (1995) Use of CAD/CAM system to fabricate dental prostheses. Part 1: CAD for a clinical crown restoration. Int J Prosthodont $8,252-258$

8. Ohm E, Silness J (1978) The convergence angle in teeth prepared for artificial crowns. J Oral Rehabil 5, 371-375

9. Nordlander J, Weir D, Stoffer W, Ochi S (1988) The taper of clinical preparations for fixed prosthodontics. J Prosthet Dent 60, 148-151

10. Sato T, Al Mutawa N, Okada D, Hasegawa S (1998) 
A clinical study on abutment taper and height of full cast crown preparations. J Med Dent Sci 45, 205210

11. Kournetas N, Jaeger B, Axmann D, Groten M, Lachmann S, Weber H, Geis-Gerstorfer J (2004) Assessing the reliability of a digital preparation assistant system used in dental education. J Dent Educ 68, 1228-1234

12. Buchanan JA (2004) Experience with virtual realitybased technology in teaching restorative dental procedures. J Dent Educ 68, 1258-1265 